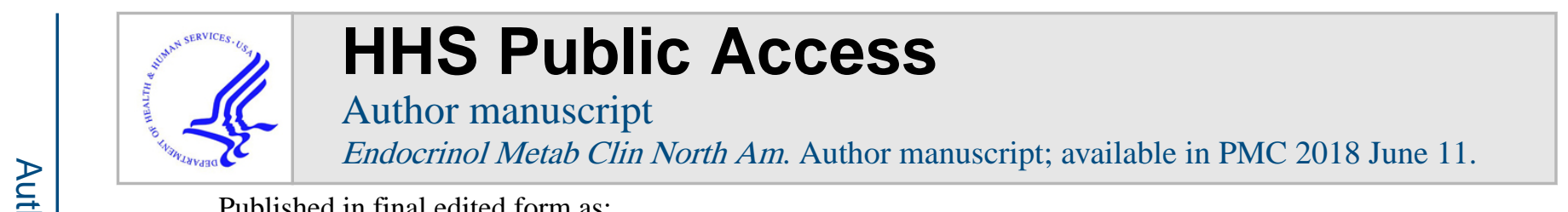

Published in final edited form as:

Endocrinol Metab Clin North Am. 2015 September ; 44(3): 649-661. doi:10.1016/j.ecl.2015.05.009.

\title{
Menopause and Sexuality
}

Kimberley Thornton, MD¹, Judi Chervenak, MD1, and Genevieve Neal-Perry, MD PhD²

${ }^{1}$ Department of Obstetrics and Gynecology, Einstein College of Medicine and Montefiore Medical Center, Bronx, NY

${ }^{2}$ Department of Obstetrics and Gynecology, University of Washington, Seattle, WA

\section{Synopsis}

Sexuality is an important component in the lives of menopausal women. The addition of phosphodiesterase type 5 inhibitors for the treatment of male erectile dysfunction in combination with longer life expectancy has impacted attitudes and expectations to maintain sexual functioning. Despite the importance of sexual function in menopausal women, sexual dysfunction increases with age. Age-related decline in sexual function may significantly reduce quality of life, making recognition of sexual dysfunction by physicians important for getting menopausal women effective care. Sexual dysfunction can result from multiple etiologies including psychosocial factors, medication side effects, vulvovaginal atrophy, chronic illness, or hypoactive sexual desire disorder (HSDD). Discovering the etiology and identifying modifiable factors of the sexual function will help define appropriate treatment.

\section{Keywords}

Menopause; sexuality; vulvovaginal atrophy; hypoactive sexual desire disorder; hormone therapy

\section{Introduction}

Sexuality may impact quality of life through effects on the emotional and psychological health of a woman. Consequently, clinicians who take care of women appreciate when they may be vulnerable to sexual dysfunction. The menopausal transition, a time characterized by hormonal, physiological and social changes, is often associated with sexual dysfunction. The physiological mechanism by which the menopausal transition affect sexual health involves declining and fluctuating gonadal steroid hormone levels which adversely affect elasticity of the vaginal mucosa, vaginal secretions and result in vaginal atrophy and pain with sexual intercourse [1]. Additionally, social conditions or life stressors such as divorce, lack of a partner, job loss, or declining health may affect desire for sexual intercourse.

Corresponding author: Genevieve Neal-Perry, MD PhD, Department of Obstetrics and Gynecology, University of Washington, Seattle, WA, 1959 NE Pacific Street, Box 356460, Seattle, WA 98195-6460, NealPerr@uw.edu.

Publisher's Disclaimer: This is a PDF file of an unedited manuscript that has been accepted for publication. As a service to our customers we are providing this early version of the manuscript. The manuscript will undergo copyediting, typesetting, and review of the resulting proof before it is published in its final citable form. Please note that during the production process errors may be discovered which could affect the content, and all legal disclaimers that apply to the journal pertain. 
Improved access to medical care and nutrition has increased the average life expectancy. Therefore, the average woman making the transition into menopause can expect to live for at least 25 years [2]. With increased expectations for a longer and healthier life, women are thinking more about quality of life issues, which include maintaining sexual function [3]. Additionally, attitudes and expectations regarding sexual function were further impacted when the FDA approved phosphodiesterase type 5 inhibitors for male erectile dysfunction, which resulted in more menopausal women with male partners who have renewed sexual interest and improved function [4, 5].

\section{Attitudes About Sex and the Menopause}

Regardless of age and menopausal status, sexual interest continues for many women. Seventy six percent of middle-aged women in the Study of Women's Health Across the Nation (SWAN) reported sex was moderately or extremely important to them [6]. Even though sex is important to reproductively senescing women, sexual activity and function decline with age. In the Women's Healthy Ageing Project (WHAP) cohort, an extension of the Melbourne Women's Midlife Health Project, a significant decline from 74 to 56\% in sexual activity $(\mathrm{p}<0.001)$ was reported between early postmenopausal women and late postmenopausal women [7]. Short Personal Experience Questionnaire (SPEQ; a 9-item sexual-function instrument) scores also indicated that $42 \%$ of early perimenopausal women had sexual dysfunction in the Melbourne Women's Midlife Health Project at baseline. After eight years of follow-up, the percent of women with sexual dysfunction, as determined by SPEQ scores, more than doubled to $88 \%$ [8]. The etiology of this decline in sexual function and activity may vary and is often multifactorial. Thus, a careful evaluation is required to determine the cause and recommend the best intervention.

\section{Physiology}

In the regularly menstruating woman, each month follicular phase follicle stimulating hormone (FSH) stimulates follicular growth and estradiol synthesis. Increasing estradiol production from the dominant follicle mediates a negative feedback and suppressive effect on FSH and luteinizing hormone (LH). Estradiol synthesis from the dominant follicle continues until a critical level is reached and estradiol positive feedback induces positive feedback, an LH surge and ovulation [9]. Estradiol synthesis during the menstrual cycle affects vaginal secretions and the vaginal mucosa.

\section{Pathophysiology}

Multiple physiologic changes that occur during the menopausal transition result from reduced ovarian reserve and reduced numbers of gonadotropin responsive follicles. Menstrual cycles in late perimenopausal women are characterized by increased FSH, decreased inhibin B, and irregularly short and long cycle lengths[10]. Until the time of the last menstrual period (LMP), estradiol levels are equally variable in perimenopausal women. By the time of the LMP, women enter a persistent state of hypogonadism and hypergonadotropism (elevated FSH and LH) [11, 12]. After estradiol falls, estrone, primarily generated by the aromatization of androgens, becomes the main circulating estrogen. 
Compared to estradiol, serum androgen levels demonstrate a steady but less dramatic decline (Figure 1) $[8,13]$. The less dramatic fall in serum androgens is related to the decrease in sex hormone binding globulin associated with hypoestrogenism[14].

\section{Sex and Hormones}

Hormonal changes during menopause may impact sexual functioning. A prospective, population-based study of Australian born women, observed for eight years as they passed through natural menopause, reported that low estrogen levels adversely affected sexual interest and responsiveness, but did not affect the frequency of sexual activity [15]. Total testosterone was not significantly affected in this cohort. Moreover, free testosterone levels did not significantly affect any sexual domains. Similarly, no significant difference in testosterone levels was observed in women undergoing natural menopause with SPEQ scores suggesting sexual dysfunction compared to women with SPEQ scores showing no dysfunction [8]. It is worth noting that it is possible that there are differences in serum testosterone, however, it may be difficult to observe significant differences in very low levels of testosterone because of the sensitivity limitations of non-mass spectrometry-based testosterone assays [8].

Pelvic organ prolapse (POP) is the descent of one or more of the following: anterior vaginal wall, posterior vaginal wall, uterus or apex of the vagina. The incidence of pelvic floor weakening increases with aging and is thought to result from a combination of connective tissue degradation, pelvic denervation, and devascularization, all of which predispose to prolapse [16]. Dyspareunia, chronic pelvic pain, and modified self-image are associated with POP. Any one of these adverse symptoms can devastate sexual function.

\section{Hypoactive Sexual Desire Disorder}

Hypoactive sexual desire disorder (HSDD) occurs when there is a persistent or recurrent absence of sexual fantasies or desire for sexual activity that results in personal distress. For the diagnosis of HSDD to be made, iatrogenic or organic causes for sexual dysfunction must be ruled out and the patient must report marked distress or interpersonal difficulty [17]. Recently, the Diagnostic and Statistical Manual of Mental Disorders Fifth Addition (DSMV) grouped HSDD with female sexual arousal disorder (FSAD) under the category of female sexual interest/ arousal disorders (FSIAD) [18]. The DSM-V criteria for the diagnosis of FSIAD is shown in Box 1.

The prevalence of HSDD has been difficult to determine and has varied among studies. Disparate estimates of prevalence may reflect differences in the age of the study group and different criteria for diagnosis and study inclusion. The Prevalence of Female Sexual Problems Associated with Distress and Determinants of Treatment Seeking (PRESIDE) survey of 31,581 US women 18 years or older found $8.9 \%$ of women aged 18 to 44 years, $12.3 \%$ of women aged 45 to 64 years, and $7.4 \%$ of women older than 65 years had low desire and distress [19]. The Global Study of Sexual Attitudes and Behavior, reported a 25 to $43 \%$ prevalence of symptoms consistent with lack of sexual interest in women[20]. These numbers represent all women reporting occasional, periodic, or frequent problems with 
desire. If the population were restricted to women reporting frequent problems the prevalence of low desire would vary between $5.4 \%$ and $13.6 \%$ [21].

The risk for HSDD is greatest in women who have undergone surgical menopause. It is hypothesized that abrupt reductions in circulating 17-B-estradiol and testosterone levels significantly contribute to HSDD because testosterone replacement can increase sexual desire and arousal in this group of women [22] [23]. The Women's International Study of Health and Sexuality (WISHeS) reported surgically-induced menopausal women 20 to 49 years old had significantly higher rates of HSDD compared to gonadally intact, age-matched and regularly cycling control women ( $26 \%$ vs. $14 \%$ respectively, $\mathrm{p}=0.002)$ [24]. In contrast, no significant difference was found in the diagnosis of HSDD between surgically or naturally postmenopausal women aged 50 to 70 years ( $14 \%$ and $9 \%$ respectively, $\mathrm{p}=0.067$ ). These data suggest that healthcare providers should routinely assess women with surgical menopause for signs of sexual dysfunction. Androgen therapy may be considered in this population of women.

HSDD correlates with low feelings of physical and emotional satisfaction, poor self-image, and unhappiness [25]. Research suggests multiple psychological reasons why women choose to engage in sexual activity, including wanting to feel close to a partner, expression of love, and wanting to feel feminine[26]. When a diagnosis of HSDD is suspected, psychosocial factors such as conflict with a partner, history of sexual abuse, and cultural/religious factors should be considered, as they can significantly affect sexual desire [27].

\section{Symptomatic Vulvovaginal Atrophy}

Decreased levels of estrogen are associated with symptomatic vulvovaginal atrophy, a condition characterized by thin, pale, and dry vaginal and vulvar surfaces. During menopause, the decline in estrogen leads to a decrease in lactobacilli, acid producing bacteria that play a key role in keeping the vaginal epithelial $\mathrm{pH}$ in the range of 3.8 to 4.5 [1]. As lactobacilli decrease, the vaginal epithelium becomes more basic, leading to a change in vaginal flora. It has been noted that increased bacterial diversity is correlated with increased symptoms of vaginal dryness [28].

Symptomatic vulvovaginal atrophy is highly prevalent in midlife and menopausal women, with as many as $45 \%$ of postmenopausal women affected [29]. Symptomatic vulvovaginal atrophy is often accompanied by diminished secretions from sebaceous glands and reduced vaginal lubrication during sexual stimulation [1]. Women with vulvovaginal atrophy experience pain with intercourse that leads to decreased interest and frank avoidance of sexual activity [30]. In the Real Women's Views on Treatment Options for Menopausal Vaginal Changes (REVIVE) study, 63\% of women with symptomatic vulvovaginal atrophy reported that their symptoms interfered with enjoyment of sexual intercourse and $47 \%$ of partnered women indicated it interfered with their relationship [31, 32]. Twelve percent of women without a partner reported that they were not seeking a sexual partner due to symptoms related to vulvovaginal atrophy. Similarly, in the Vaginal Health: Insights, Views, \& Attitudes (VIVA) study, 75\% of women reported that vaginal discomfort negatively affected their sex life [33]. Despite the high prevalence of symptomatic vulvovaginal atrophy 
in midlife and postmenopausal women, almost half of women report that they never discuss the impact of the symptoms on their quality of life with a health care provider. Equally worrisome, only $7 \%$ reported that their healthcare provider ever initiated a conversation about vulvovaginal atrophy $[1,31,32]$. Health care providers should be vigilant about asking patients about satisfaction with their sex life and they should query patients about signs and symptoms of vulvovaginal atrophy.

\section{Chronic diseases and sexual dysfunction}

When a diagnosis of sexual dysfunction is suspected, a complete and detailed medical history should be obtained to evaluate women for chronic diseases that can adversely affect sexual health. This is especially true in menopause, because as women age they are at an increased risk for acquiring chronic diseases that impact sexual function [22]. Chronic diseases such as hypertension, diabetes, depression, neurological diseases, urinary incontinence, and osteoarthritis commonly impact sexual function [22, 27, 34]. Some reports suggest that cardiovascular disease impacts women's sexual desire and arousal through effects on systemic blood flow. The mechanisms by which cardiovascular disease affects sexual arousal is hypothesized to be related to the fact that female genital arousal is achieved when the vascular system increases blood flow and engorges the labia via vasodilation. Vascular disease may reduce vulvovaginal vasodilation and reduce sexual arousal [35]. Reduced physical function in obese women and women with osteoarthritis may also adversely affect sexual activity. Additionally, sexual dysfunction observed in women with diabetes is attributed to reduced energy, altered body image, and suboptimal vaginal engorgement during orgasm [22].

\section{Medications}

Medications must be considered as a possible source of sexual dysfunction in menopausal women. Organ systems have limited homeostatic reserve with aging, resulting in decreased clearance and enhanced toxicity of many drugs [36]. For these reasons, undesired effects of medications are more prevalent in the elderly. Medications commonly associated with sexual dysfunction include serotonin reuptake inhibitors (SSRIs) neuroleptics, and cardiovascular medications [22,34]. A detailed list is shown in Box 2. The menopausal transition and early postmenopausal years are considered windows of vulnerability for depression; therefore antidepressants may be a common medication amongst menopausal women [37]. SSRIs are associated with HSDD. When possible, the medication suspected of causing sexual side effects should be stopped or switched.

\section{Quality of Life Impact on Menopausal Women}

Menopausal symptoms and sexual dysfunction can negatively impact quality of life for women. A higher sense of purpose in life is reported by midlife women who report higher levels of enjoyment with sexual activity [38]. Women more likely to engage in partnered, intimate sexual activities tend to be of younger age, lower body mass index, married, and have better emotional well-being [38]. This raises the possibility that aging women affected by obesity or single status may be at high risk for experiencing an overall decrease in quality 
of life. By counseling patients about the benefits of healthy diet and exercise for weight control and screening for medications and illnesses that impact sexual function, health care providers may improve overall quality of life.

Educational level and economic status impact sexual functioning and quality of life for women. Baseline data from the SWAN study suggested that women reporting financial strain were more likely to report decreased frequency of sexual desire and arousal as well as lower levels of emotional and physical satisfaction with intercourse when compared to their more financially secure counterparts [6]. Despite their decrease in desire and arousal, the majority of these women still reported that sex was moderately to extremely important to them. Menopausal women with some college or graduate school education have better physical and mental parameters of health that are related to quality of life compared to those with a high school education or less [3]. This demonstrates that multiple socioeconomic factors impact sexual functioning and quality of life for menopausal women. Women affected by socioeconomic stressors may benefit from increased social support.

Menopausal symptoms such as vasomotor symptoms and vaginal dryness negatively impact health related quality of life [39]. Women with vaginal dryness, even if they do not identify the symptom as bothersome, have worse mental health composite scores as well as worse emotional well-being and social functioning [3]. These data suggest that there is a need for physician vigilance and early detection so that interventions can be employed to prevent potentially debilitating effects on quality of life.

\section{Treatments}

\section{Psychological counseling}

Psychological counseling, solely or in combination with medical treatment, can be helpful to women and couples suffering from sexual dysfunction. Psychosexual therapy with the menopausal woman alone or couples' sexual therapy can be considered. Couples therapy can help identify relationship issues contributing to sexual problems and allow opportunities for couples to improve communication[40]. Issues such as relationship distress, extended periods of sexual abstinence, sexual abuse history, lack of sleep, and taking care of an elderly parent can all impact sexual function [41]. Behavioral exercises may help individuals reduce anxiety associated with sexual dysfunction [42].

\section{Estrogen}

The role of hormone therapy in consistently increasing sexual desire or activity has not been established [25]. Sexual activity analysis from the Women's Health Initiative found no statistically significant correlation between use of hormone therapy and the continuation of sexual activity, further supporting that estrogen therapy does not increase libido [46]. Current evidence does not support the use of estrogen or combined estrogen and progesterone therapy to treat sexual interest or arousal disorders in menopausal women [4345]. Prior sexual functioning and relationship factors, such as having an attractive and available partner, a safe environment, and self-esteem, have been shown to be more important than hormonal determinants of sexual function in midlife women [15]. However, 
hormone therapy with estrogen may improve sexual function by increasing vaginal lubrication and reducing dyspareunia in women affected by vulvovaginal atrophy [25]. Local vaginal estrogen therapy is recommended as the treatment of choice for symptomatic vulvovaginal atrophy [47]. Low dose vaginal estrogen formulations come in vaginal creams, tablets, and rings (see Table 1). Systemic absorption is low for these preparations and they are not effective for the relief of vasomotor symptoms [1]. A progestin is generally not needed when low dose vaginal estrogen formulations are used, although clinical trial data supporting endometrial safety beyond 1 year are lacking [48]. Thorough evaluation of any uterine bleeding should be done in women using low dose local estrogen therapy. For women who do not want hormonal therapy, water-based lubricants and moisturizers are available over the counter to help alleviate symptoms.

\section{Testosterone}

There are currently no FDA approved testosterone formulations for the treatment of low sexual function in women. Despite this fact, many providers still prescribe compounded testosterone or use testosterone products off-label for this purpose. The use of testosterone to treat female sexual function is counterintuitive; free and total serum testosterone levels are not related to the scores of female sexual function assessment tests [49]. In contrast, multiple randomized, controlled trials have shown promising evidence that testosterone improves sexual desire and function in some menopausal women. As discussed earlier, women who undergo surgical menopause have an approximately 50\% decrease in serum testosterone and may benefit from testosterone therapy. In a randomized, double-blind, placebo-controlled trial, Shifren and colleagues demonstrated that a 300 ug transdermal testosterone patch along with conjugated equine estrogens in women with oophorectomy and hysterectomy significantly increased scores for frequency and pleasure of sexual activity compared to placebo [50]. It is important to note that several studies have now demonstrated improved sexual function with few side effects in surgically, as well as naturally menopausal women with use of the $300 \mathrm{ug}$ transdermal testosterone patch[22, 34, 51]. However, clinicians must seriously consider and discuss the risk and benefits of testosterone therapy in a woman who undergoes natural menopause.

Side effects of testosterone treatment tend to be mild, and commonly include application site reactions and facial hair. Statistically significant benefits of treatment are not observed until after 4 to 16 weeks of therapy, suggesting that testosterone therapy may require several months before a full assessment of benefit can be made. This is an important point to stress when counseling patients on expectations of therapy. Oral testosterone therapy increases triglycerides and low-density lipoprotein cholesterol and reduces high-density lipoprotein cholesterol. These adverse lipid effects have raised concern regarding safety [52]. In 967 surgically menopausal women who were also on estrogen replacement, transdermal testosterone therapy did not affect lipid profiles over 4 years of follow up [53]. This suggests that the route of administration affects whether or not testosterone impacts the lipid profile and suggests that transdermal testosterone is preferred over oral formulations.

Estrogen is known to increase SHBG levels and suppress luteinizing hormone secretion, decreasing testosterone availability and ovarian androgen synthesis [54]. These effects of 
estrogen prompted studies designed to investigate the side effects of testosterone therapy without estrogen in menopausal women. A randomized, double-blinded, placebo controlled trial in both surgically and naturally menopause women showed that the $300 \mathrm{ug}$ testosterone patch modestly improved the mean frequency of satisfying sexual episodes to 2.1 episodes per month compared with 0.7 in the placebo group [55]. There was no significant difference in satisfying sexual episodes in those using a $150 \mathrm{ug}$ testosterone patch, indicating that the $300 \mathrm{ug}$ dose is needed for a clinical effect. As seen in other studies, a higher incidence of hair growth was seen in the testosterone-treated women. It was also noted that there were three cases of breast cancer reported in the testosterone treated women in this study and more cases of vaginal bleeding. As these women were not on estrogen therapy, the increase in bleeding could possibly result from atrophic endometrium. Although no serious endometrial disease was found in the treatment group, the long-term safety profile of testosterone therapy without estrogen still needs additional investigation. If testosterone therapy is to be initiated for sexual dysfunction, it is generally recommended that treatment not exceed six months and that a patch is used.

\section{Future Directions}

Lorexys is a potential new medication for hypoactive sexual desire disorder currently under investigation. Lorexys is an oral, non-hormonal medication that works by balancing the dopamine, serotonin, and norepinephrine neurotransmitters that regulate sexual inhibition and sexual excitation [49]. It is a combination of two antidepressants already on the market: bupropion and trazodone. It is currently in a Phase 2 a clinical study.

Flibanserin is another non-hormonal medication under investigation for treatment of HSDD. Flibanserin is a postsynaptic agonist of serotonin 5-HT receptor $1 \mathrm{~A}$ and an antagonist of serotonin $5 \mathrm{HT}$ receptor $2 \mathrm{~A}$ that has been shown to induce transient decreases in serotonin and increases in dopamine and norepinephrine in certain regions of the brain [56]. The SNOWDROP trial, a multicenter, randomized, double-blinded, placebo controlled trial of 949 naturally menopausal women, found significant improvement in sexual desire and satisfying sexual events in postmenopausal women using flibanserin compared to placebo [57]. The most common side effects of flibanserin were dizziness, insomnia, nausea, and headache. Although flibanserin has been studied in both premenopausal and postmenopausal women, its application to the FDA is only for premenopausal women.

\section{Conclusion}

Sexual health and function are essential components in the care of menopausal women. Most menopausal women consider sex to be an important part of their life and strongly desire to maintain a robust sexual life. However, the risk of acquiring a co-morbidity that adversely affects sexual satisfaction and function as well as the risk for using medication that affects sexual function increases as women age. Although sexual dissatisfaction and dysfunction are highly prevalent in perimenopausal and postmenopausal women, few disclose their concerns to the healthcare provider. Thus, healthcare providers should be proactive and routinely query perimenopausal and menopausal patients about their satisfaction with sex and their sexual functioning. If sexual dissatisfaction or dysfunction is suspected, then a full medical 
and social history with focused question about factors that affect sexual function should be undertaken. Questions about living situations should be fully explored because menopause often coincides with life-stressing events such as children leaving the home, sick parents, or loss of a partner. Discovering the etiology and identifying modifiable factors the influence sexual function will help define appropriate treatment. Finally, sexual health in menopausal women and their partners is important. Age-related declines in sexual function may significantly reduce quality of life. Increased recognition by physicians and validation of patient concerns as well as expanded discussions about sexual dysfunction with patients may offer an opportunity for effective intervention and improve the quality of life for affected women.

\section{References}

1. Management of symptomatic vulvovaginal atrophy: 2013 position statement of The North American Menopause Society. Menopause. 2013; 20(9):888-902. quiz 903-4. [PubMed: 23985562]

2. Sherry Murphy JX, Kenneth Kochanek. Deaths: Final Data for 201. NVS Report, Editor. 2010

3. Hess R, et al. The impact of menopause on health-related quality of life: results from the STRIDE longitudinal study. Qual Life Res. 2012; 21(3):535-44. [PubMed: 21755412]

4. Potts A, et al. The downside of Viagra: women's experiences and concerns. Sociol Health Illn. 2003; 25(7):697-719. [PubMed: 19774744]

5. Barnett ZL, Robleda-Gomez S, Pachana NA. Viagra: the little blue pill with big repercussions. Aging Ment Health. 2012; 16(1):84-8. [PubMed: 21767227]

6. Cain VS, et al. Sexual functioning and practices in a multi-ethnic study of midlife women: baseline results from SWAN. J Sex Res. 2003; 40(3):266-76. [PubMed: 14533021]

7. Lonnee-Hoffmann RA, et al. Sexual Function in the Late Postmenopause: A Decade of Follow-Up in a Population-Based Cohort of Australian Women. J Sex Med. 2014

8. Dennerstein L, et al. Hormones, mood, sexuality, and the menopausal transition. Fertil Steril. 2002; 77(Suppl 4):S42-8. [PubMed: 12007901]

9. Sherman BM, Korenman SG. Hormonal characteristics of the human menstrual cycle throughout reproductive life. J Clin Invest. 1975; 55(4):699-706. [PubMed: 1120778]

10. Santoro N, Chervenak JL. The menopause transition. Endocrinol Metab Clin North Am. 2004; 33(4):627-36. [PubMed: 15501637]

11. Santoro N, et al. Characterization of reproductive hormonal dynamics in the perimenopause. J Clin Endocrinol Metab. 1996; 81(4):1495-501. [PubMed: 8636357]

12. Burger HG, et al. Cycle and hormone changes during perimenopause: the key role of ovarian function. Menopause. 2008; 15(4 Pt 1):603-12. [PubMed: 18574431]

13. Rothman MS, et al. Reexamination of testosterone, dihydrotestosterone, estradiol and estrone levels across the menstrual cycle and in postmenopausal women measured by liquid chromatographytandem mass spectrometry. Steroids. 2011; 76(1-2):177-82. [PubMed: 21070796]

14. Burger HG, et al. A prospective longitudinal study of serum testosterone, dehydroepiandrosterone sulfate, and sex hormone-binding globulin levels through the menopause transition. J Clin Endocrinol Metab. 2000; 85(8):2832-8. [PubMed: 10946891]

15. Dennerstein L, Lehert $P$, Burger $H$. The relative effects of hormones and relationship factors on sexual function of women through the natural menopausal transition. Fertil Steril. 2005; 84(1): 174-80. [PubMed: 16009174]

16. Mannella P, et al. The female pelvic floor through midlife and aging. Maturitas. 2013; 76(3):230-4. [PubMed: 24055286]

17. Brotto LA. The DSM diagnostic criteria for hypoactive sexual desire disorder in women. Arch Sex Behav. 2010; 39(2):221-39. [PubMed: 19777334]

18. Diagnostic and Statistical Manual of Mental Disorders. 5. American Psyciatric Association; Washington, DC: 2013. 
19. Shifren JL, et al. Sexual problems and distress in United States women: prevalence and correlates. Obstet Gynecol. 2008; 112(5):970-8. [PubMed: 18978095]

20. Laumann EO, et al. Sexual problems among women and men aged 40-80 y: prevalence and correlates identified in the Global Study of Sexual Attitudes and Behaviors. Int J Impot Res. 2005; 17(1):39-57. [PubMed: 15215881]

21. Segraves R, Woodard T. Female hypoactive sexual desire disorder: History and current status. J Sex Med. 2006; 3(3):408-18. [PubMed: 16681466]

22. Ambler DR, Bieber EJ, Diamond MP. Sexual function in elderly women: a review of current literature. Rev Obstet Gynecol. 2012; 5(1):16-27. [PubMed: 22582123]

23. Davison SL, et al. Androgen levels in adult females: changes with age, menopause, and oophorectomy. J Clin Endocrinol Metab. 2005; 90(7):3847-53. [PubMed: 15827095]

24. Leiblum SR, et al. Hypoactive sexual desire disorder in postmenopausal women: US results from the Women's International Study of Health and Sexuality (WISHeS). Menopause. 2006; 13(1):4656. [PubMed: 16607098]

25. Nappi RE, Wawra K, Schmitt S. Hypoactive sexual desire disorder in postmenopausal women. Gynecol Endocrinol. 2006; 22(6):318-23. [PubMed: 16785156]

26. Meston CM, Buss DM. Why humans have sex. Arch Sex Behav. 2007; 36(4):477-507. [PubMed: 17610060]

27. Kingsberg SA, Rezaee RL. Hypoactive sexual desire in women. Menopause. 2013; 20(12):1284300. [PubMed: 24219879]

28. Hummelen R, et al. Vaginal microbiome and epithelial gene array in post-menopausal women with moderate to severe dryness. PLoS One. 2011; 6(11):e26602. [PubMed: 22073175]

29. Santoro N, Komi J. Prevalence and impact of vaginal symptoms among postmenopausal women. J Sex Med. 2009; 6(8):2133-42. [PubMed: 19493278]

30. Tan O, Bradshaw K, Carr BR. Management of vulvovaginal atrophy-related sexual dysfunction in postmenopausal women: an up-to-date review. Menopause. 2012; 19(1):109-17. [PubMed: 22011753]

31. Wysocki S, Kingsberg S, Krychman M. Management of Vaginal Atrophy: Implications from the REVIVE Survey. Clin Med Insights Reprod Health. 2014; 8:23-30. [PubMed: 24987271]

32. Kingsberg SA, et al. Vulvar and vaginal atrophy in postmenopausal women: findings from the REVIVE (REal Women's VIews of Treatment Options for Menopausal Vaginal ChangEs) survey. J Sex Med. 2013; 10(7):1790-9. [PubMed: 23679050]

33. Simon JA, et al. Vaginal health in the United States: results from the Vaginal Health: Insights, Views \& Attitudes survey. Menopause. 2013; 20(10):1043-8. [PubMed: 23571518]

34. Buster JE. Managing female sexual dysfunction. Fertil Steril. 2013; 100(4):905-15. [PubMed: 24074537]

35. Nascimento ER, et al. Sexual dysfunction and cardiovascular diseases: a systematic review of prevalence. Clinics (Sao Paulo). 2013; 68(11):1462-8. [PubMed: 24270960]

36. Camacho ME, Reyes-Ortiz CA. Sexual dysfunction in the elderly: age or disease? Int J Impot Res. 2005; 17(Suppl 1):S52-6. [PubMed: 16391544]

37. Soares CN. Mood disorders in midlife women: understanding the critical window and its clinical implications. Menopause. 2014; 21(2):198-206. [PubMed: 24448106]

38. Prairie BA, et al. A higher sense of purpose in life is associated with sexual enjoyment in midlife women. Menopause. 2011; 18(8):839-44. [PubMed: 21659908]

39. Avis NE, et al. Change in health-related quality of life over the menopausal transition in a multiethnic cohort of middle-aged women: Study of Women's Health Across the Nation. Menopause. 2009; 16(5):860-9. [PubMed: 19436224]

40. Althof SE. Sex therapy and combined (sex and medical) therapy. J Sex Med. 2011; 8(6):1827-8. [PubMed: 21631735]

41. Goldstein I. Current management strategies of the postmenopausal patient with sexual health problems. J Sex Med. 2007; 4(Suppl 3):235-53. [PubMed: 17394596]

42. Al-Azzawi F, et al. Therapeutic options for postmenopausal female sexual dysfunction. Climacteric. 2010; 13(2):103-20. [PubMed: 19958161] 
43. Modelska K, Cummings S. Female sexual dysfunction in postmenopausal women: systematic review of placebo-controlled trials. Am J Obstet Gynecol. 2003; 188(1):286-93. [PubMed: $12548231]$

44. Alexander JL, et al. The effects of postmenopausal hormone therapies on female sexual functioning: a review of double-blind, randomized controlled trials. Menopause. 2004; 11(6 Pt 2): 749-65. [PubMed: 15543027]

45. Wierman ME, et al. Endocrine aspects of women's sexual function. J Sex Med. 2010; 7(1 Pt 2): 561-85. [PubMed: 20092453]

46. Gass ML, et al. Patterns and predictors of sexual activity among women in the Hormone Therapy trials of the Women's Health Initiative. Menopause. 2011; 18(11):1160-71. [PubMed: 21983008]

47. North American Menopause, S. The 2012 hormone therapy position statement of: The North American Menopause Society. Menopause. 2012; 19(3):257-71. [PubMed: 22367731]

48. Sturdee DW, Panay N, G. International Menopause Society Writing. Recommendations for the management of postmenopausal vaginal atrophy. Climacteric. 2010; 13(6):509-22. [PubMed: 20883118]

49. Davis SR, et al. Circulating androgen levels and self-reported sexual function in women. JAMA. 2005; 294(1):91-6. [PubMed: 15998895]

50. Shifren JL, et al. Transdermal testosterone treatment in women with impaired sexual function after oophorectomy. N Engl J Med. 2000; 343(10):682-8. [PubMed: 10974131]

51. Shifren JL, et al. Testosterone patch for the treatment of hypoactive sexual desire disorder in naturally menopausal women: results from the INTIMATE NM1 Study. Menopause. 2006; 13(5): 770-9. [PubMed: 16932240]

52. Maclaran K, Panay N. The safety of postmenopausal testosterone therapy. Womens Health (Lond Engl). 2012; 8(3):263-75. [PubMed: 22554174]

53. Nachtigall L, et al. Safety and tolerability of testosterone patch therapy for up to 4 years in surgically menopausal women receiving oral or transdermal oestrogen. Gynecol Endocrinol. 2011; 27(1):39-48. [PubMed: 21142609]

54. Casson PR, et al. Effect of postmenopausal estrogen replacement on circulating androgens. Obstet Gynecol. 1997; 90(6):995-8. [PubMed: 9397118]

55. Davis SR, et al. Testosterone for low libido in postmenopausal women not taking estrogen. N Engl J Med. 2008; 359(19):2005-17. [PubMed: 18987368]

56. Borsini F, et al. Pharmacology of flibanserin. CNS Drug Rev. 2002; 8(2):117-42. [PubMed: 12177684]

57. Simon JA, et al. Efficacy and safety of flibanserin in postmenopausal women with hypoactive sexual desire disorder: results of the SNOWDROP trial. Menopause. 2014; 21(6):633-40. [PubMed: 24281236] 


\section{Key points}

- Sexual dysfunction increases with age and is highly prevalent amongst menopausal women.

- Most menopausal women consider sex to be an important part of their life and strongly desire to maintain sexual activity.

- Few women disclose their concerns to healthcare providers; therefore, healthcare providers should routinely query perimenopausal and menopausal patients about their satisfaction with their sexual functioning. 


\section{Box 1}

\section{DSM 5: Female sexual interest/ arousal disorder}

A. Lack of, or significantly reduced, sexual interest/ arousal, as manifested by at least three of the following:

a. Absent/ reduced interest in sexual activity

b. Absent/ reduced sexual/ erotic thoughts or fantasies

c. $\quad \mathrm{No} /$ reduced initiation of sexual activity and unreceptive to partner's attempts to initiate

d. Absent/ reduced sexual excitement/ pleasure during sexual activity in almost all or all (75-100\%) sexual encounters

e. Absent/ reduced sexual interest/ arousal in response to any internal or external sexual/ erotic cues (written, verbal, or visual)

f. Absent/ reduced genital or nongenital sensations during sexual activity in almost all or all (75-100\%) sexual encounters

B. The symptoms in Criterion A have persisted for a minimum duration of approximately 6 months.

C. The symptoms in Criterion A cause clinically significant distress in the individual.

D. The sexual dysfunction is not better explained by a nonsexual mental disorder or as a consequence of severe relationship distress or other significant stressors and is not attributable to the effects of a substance/medication or another medical condition

From Diagnostic and Statistical Manual of Mental Disorders, 5th Edition. 2013, American Psyciatric Association: Washington, DC, with permission. 
Box 2

Medications Associated with Sexual Dysfunction

Antidepressants/ Mood stabilizers

Selective serotonin reuptake inhibitors

Tricyclic antidepressants

Monoamine oxidase inhibitors

Benzodiazapines

Lithium

Antipsychotics

Cardiovascular medications

Beta blockers

Digoxin

Antilipid medications

Spironolactone

Hormonal preparations

Oral contraceptives

GnRH agonists

Antiandrogens

Neuroleptic medications

Steroids

Antiepileptics

Antihistamines

Anticholinergics 


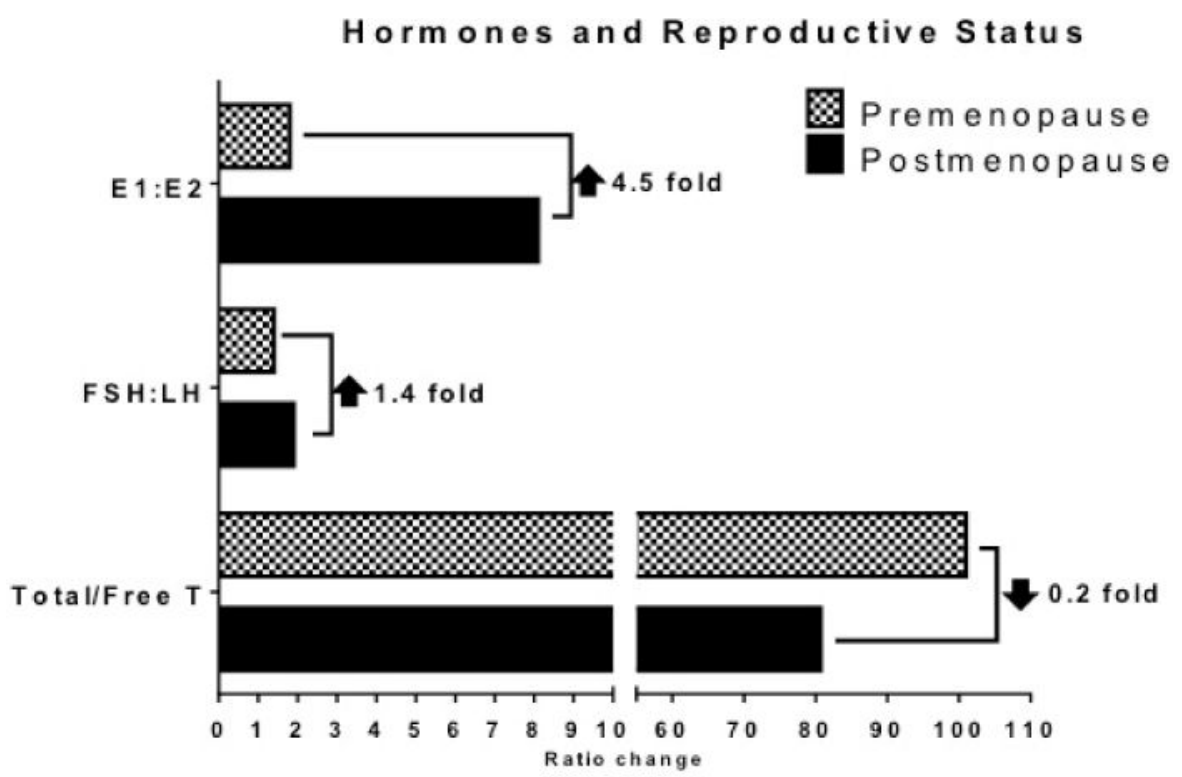

Figure 1. Characteristic hormone changes in the menopausal as compared to premenopausal women

Compared to premenopausal women, menopausal women experience significant shifts in serum levels of gonadal steroids and gonadotropin. Reduced ovarian estradiol synthesis results in a 4.5-fold increase in the estrone to estradiol and 1.4-fold increase in the FSH to LH values in menopausal women. Menopausal women synthesize 0.2-fold less androgens than premenopausal women. However, the fold change is not nearly as great as that observed in estradiol.

Data from Rothman, M.S., et al., Reexamination of testosterone, dihydrotestosterone, estradiol and estrone levels across the menstrual cycle and in postmenopausal women measured by liquid chromatography-tandem mass spectrometry. Steroids, 2011. 76(1-2): p. 177-82. 


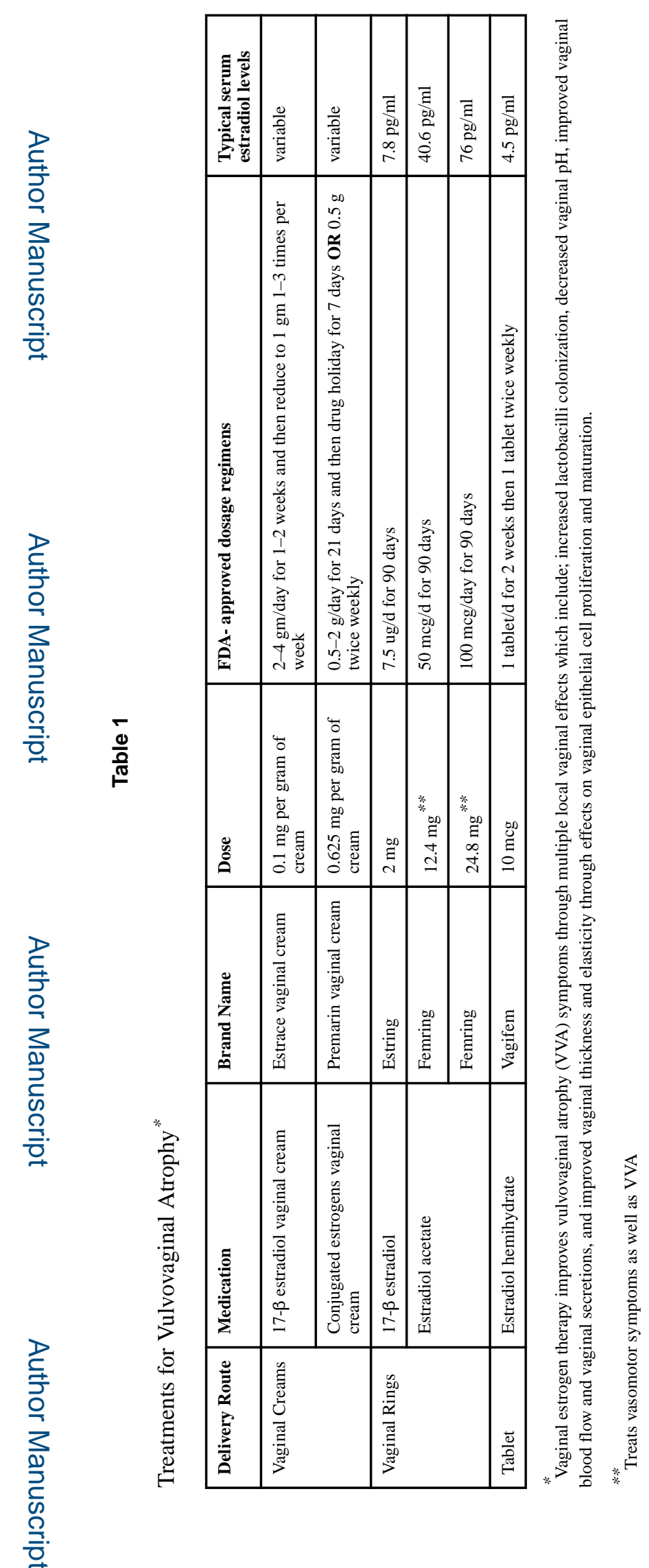

Endocrinol Metab Clin North Am. Author manuscript; available in PMC 2018 June 11. 